\title{
Do changes in cardiovascular risk factors explain the increasing socioeconomic difference in mortality from ischaemic heart disease in Finland?
}

Erkki Vartiainen, Juha Pekkanen, Seppo Koskinen, Pekka Jousilahti, Veikko Salomaa, Pekka Puska

\begin{abstract}
Study objective-To estimate the extent to which changes in blood pressure, smoking, and serum cholesterol concentration explain the observed increase in socioeconomic differences in mortality from ischaemic heart disease (IHD) in Finland during the past 20 years.

Design-Predicted changes in mortality from IHD were calculated using logistic regression models with the risk factor levels assessed by cross sectional population surveys conducted in 1972, 1977, 1982, and 1987. The subjects included white collar and blue collar workers and farmers. The predicted changes were compared with the observed mortality changes in the same socioeconomic groups in the total population of the same geographical area. Setting-North Karelia and Kuopio provinces, eastern Finland.
\end{abstract}

Participants-16 741 men and 16389 women aged 30-59 randomly drawn from the population registers of the study areas. Mortality data were obtained from the total population in the same areas.

Main results-In men, the changes in diastolic blood pressure, total serum cholesterol, and smoking predicted a $28 \%$ decline in the mortality from IHD among white collar workers, a $30 \%$ decline among blue collar workers, and a $33 \%$ decline in farmers. Observed declines in the same socioeconomic groups were $61 \%, 40 \%$, and $37 \%$, respectively. In women, the predicted decline was $41 \%$ among white collar workers, $35 \%$ among blue collar workers, and $39 \%$ among farmers. The respective observed declines were $57 \%, 43 \%$, and $20 \%$.

Conclusions-Less than half of the decline in IHD mortality among white collar men was explained by the risk factor changes, while they explained $75 \%$ of the decline among blue collar men and $89 \%$ of the decline among male farmers. Changes in

Accepted for publication 16 September 1997

Table 1 Samples (number) and participation rates (\%) by sex, year, and area in persons aged 30-59 years

\begin{tabular}{|c|c|c|c|c|c|c|c|c|}
\hline \multirow[b]{3}{*}{ Year } & \multicolumn{4}{|c|}{ North Karelia } & \multicolumn{4}{|c|}{ Kuopio Province } \\
\hline & \multicolumn{2}{|l|}{ Men } & \multicolumn{2}{|l|}{ Women } & \multicolumn{2}{|l|}{ Men } & \multicolumn{2}{|l|}{ Women } \\
\hline & Number & $\%$ & Number & $\%$ & Number & $\%$ & Number & $\%$ \\
\hline 1972 & 1959 & 94 & 2056 & 96 & 2918 & 91 & 2949 & 94 \\
\hline 1977 & 2063 & 87 & 2020 & 91 & 2933 & 89 & 2996 & 92 \\
\hline 1982 & 1599 & 77 & 1511 & 84 & 1459 & 83 & 1143 & 88 \\
\hline 1987 & 1521 & 79 & 1485 & 87 & 762 & 82 & 744 & 87 \\
\hline
\end{tabular}

risk factors did not explain the increasing difference in IHD mortality between the socioeconomic groups, especially among men.

(F Epidemiol Community Health 1998;52:416-419)

Mortality from ischaemic heart disease (IHD) has been declining in most industrialised countries during the past 20 to 30 years. ${ }^{1}$ We have previously reported that most of the decline in IHD mortality in Finland can be explained by a decline in the classic cardiovascular risk factors, smoking, high serum cholesterol, and high blood pressure. ${ }^{2}$ Despite this positive development the relative differences in IHD mortality between socioeconomic groups have been growing in Finland. ${ }^{34}$ Several studies suggest that about half of the differences between socioeconomic groups can be explained by the main risk factors. ${ }^{5-10}$ We are not aware of any previous studies, however, that relate changes in socioeconomic differences in IHD mortality with changes in risk factor levels in a population.

The aim of this paper is to assess the extent to which the changes in blood pressure, smoking, and serum total cholesterol can explain the observed growing socioeconomic differences in IHD mortality in Finland.

\section{Methods}

Four cross sectional population surveys, conducted in 1972, 1977, 1982, and 1987, have assessed the levels of coronary risk factors in Finland's eastern provinces of North Karelia and Kuopio. ${ }^{11}$ For each survey, an independent random sample was drawn from the national population register. In the 1972 and 1977 surveys, a random sample of $6.6 \%$ of the population born during 1913-1947 was drawn in both areas. In 1982 and 1987, each area's samples were stratified by sex and 10 year age group. The age range in all four surveys was 30-59 years.

The survey methods used in 1982 and 1987 followed the WHO MONICA (Multinational MONItoring of trends and determinants in CArdiovascular disease) protocol, which were comparable with the methods used in 1972 and 1977. Blood pressure was measured from the right arm of the subject, who was seated for five minutes before the measurement. The fifth phase of the Korotkoff sounds was recorded as the diastolic pressure. In 1972 and 1977, a shorter cuff bladder $(23 \mathrm{~cm})$ was used than in 1982 and $1987(42 \mathrm{~cm})$. 
Table 2 Risk factor levels from 1972 to 1987 by socioeconomic group and sex

\begin{tabular}{|c|c|c|c|c|}
\hline & Cholesterol & $\begin{array}{l}\text { Diastolic blood } \\
\text { pressure }\end{array}$ & Smoking (\%) & Number \\
\hline \multicolumn{5}{|l|}{ Men } \\
\hline \multicolumn{5}{|c|}{ White collar } \\
\hline 1972 & 6.77 & 92.4 & 44 & 884 \\
\hline 1977 & 6.51 & 90.1 & 41 & 1169 \\
\hline 1982 & 6.17 & 87.4 & 35 & 989 \\
\hline 1987 & 6.14 & 88.2 & 33 & 644 \\
\hline \multicolumn{5}{|c|}{ Blue collar } \\
\hline 1972 & 6.90 & 92.7 & 61 & 2038 \\
\hline 1977 & 6.71 & 91.4 & 53 & 2202 \\
\hline 1982 & 6.28 & 87.8 & 49 & 1788 \\
\hline 1987 & 6.20 & 88.3 & 47 & 818 \\
\hline \multicolumn{5}{|l|}{ Farmers } \\
\hline 1972 & 7.09 & 92.0 & 48 & 1212 \\
\hline 1977 & 6.84 & 90.4 & 42 & 1007 \\
\hline 1982 & 6.42 & 87.4 & 32 & 521 \\
\hline 1987 & 6.31 & 87.5 & 30 & 281 \\
\hline \multicolumn{5}{|l|}{ Women } \\
\hline \multicolumn{5}{|c|}{ White collar } \\
\hline 1972 & 6.69 & 90.0 & 14 & 1411 \\
\hline 1977 & 6.34 & 86.3 & 14 & 1800 \\
\hline 1982 & 5.91 & 84.4 & 17 & 1543 \\
\hline 1987 & 5.80 & 82.0 & 15 & 1124 \\
\hline \multicolumn{5}{|c|}{ Blue collar } \\
\hline 1972 & 6.85 & 91.6 & 15 & 1564 \\
\hline 1977 & 6.58 & 87.9 & 16 & 1673 \\
\hline 1982 & 6.19 & 85.1 & 18 & 1298 \\
\hline 1987 & 6.02 & 84.1 & 22 & 642 \\
\hline \multicolumn{5}{|l|}{ Farmers } \\
\hline 1972 & 7.03 & 93.0 & 5 & 1455 \\
\hline 1977 & 6.65 & 88.0 & 5 & 1175 \\
\hline 1982 & 6.20 & 83.9 & 8 & 415 \\
\hline 1987 & 6.12 & 84.6 & 9 & 199 \\
\hline
\end{tabular}

In 1972 and 1977 serum cholesterol was determined from frozen samples using the Liebermann-Burchard method, whereas in 1982 and 1987 serum cholesterol was determined from fresh serum samples using an enzymatic method (CHOD-PAP, Boehringer Mannheim, Monotest). The enzymatic assay gave $2.4 \%$ lower values than the LiebermannBurchard method. The cholesterol values of the 1972 and 1977 surveys were corrected for this difference in the analyses. Cholesterol determinations were carried out in the same central laboratory standardised against national and international reference laboratories.

Smoking was assessed by a self administered questionnaire. Subjects who had smoked regularly in their life and had smoked in the past half year were regarded as smokers. Participation rates were more than $90 \%$, in both areas and in both sexes in the first survey in 1972 . The proportion participating decreased slightly over time. The lowest level of participation, $77 \%$, was observed among men in North Karelia in 1982 (table 1).

Table 3 Age standardised IHD mortality per 100000 in the study areas (95\% confidence intervals) from 1971 to 1990 for men and women aged 35-64. Based on the national cause of death data linked with census records

\begin{tabular}{lccl}
\hline & White collar workers & Blue collar workers & Farmers \\
\hline Men & & & \\
$1971-1975$ & $550(487$ to 618$)$ & $622(583$ to 663$)$ & $524(486$ to 564$)$ \\
$1976-1980$ & $417(368$ to 471$)$ & $580(542$ to 620$)$ & $459(420$ to 499$)$ \\
$1981-1985$ & $377(334$ to 423$)$ & $505(472$ to 540$)$ & $417(378$ to 459$)$ \\
$1986-1990$ & $215(186$ to 248$)$ & $376(349$ to 405$)$ & $332(295$ to 372$)$ \\
Change & $335(290$ to 372$)$ & $246(207$ to 282$)$ & $192(143$ to 235$)$ \\
$\%$ & $61(53$ to 68$)$ & $40(33$ to 45$)$ & $37(27$ to 45$)$ \\
Women & & & \\
$1971-1975$ & $77(58$ to 102$)$ & $128(109$ to 149$)$ & $86(72$ to 102$)$ \\
$1976-1980$ & $59(43$ to 79$)$ & $101(84$ to 120$)$ & $86(72$ to 103$)$ \\
$1981-1985$ & $55(41$ to 71$)$ & $87(72$ to 103$)$ & $66(52$ to 83$)$ \\
$1986-1990$ & $33(24$ to 45$)$ & $73(60$ to 88$)$ & $69(53$ to 89$)$ \\
Change & $44(27$ to 55$)$ & $55(35$ to 71$)$ & $17(-8$ to 35$)$ \\
$\%$ & $57(34$ to 71$)$ & $43(27$ to 55$)$ & $20(-9$ to 41$)$ \\
\hline
\end{tabular}

\section{KEY POINTS}

- Serum cholesterol, blood pressure, and smoking have declined in a similar way in different socioeconomic groups in Finland.

- Decline in risk factors explain mortality decline in blue collar workers but less than half of the mortality decline in white collar workers.

- Risk factor changes cannot explain the increasing socioeconomic differences in mortality from ischaemic heart diseases.

The national mortality statistics recording IHD (ICD 410-414, 8th revision) as the underlying cause of death were linked to the survey cohort examined in 1972. Deaths occurred from 1972 to 1986 were included in the prospective follow up. Logistic regression analyses were done separately for men and women. Age, baseline values of serum total cholesterol, and diastolic blood pressure were included as continuous variables, and the smoking status was dichotomised. Interactions between the risk factors were excluded from the analysis because they were not statistically significant in multivariate models. In addition, including the years of education in the model had very little effect on the odds ratios of the risk factors.

All variables were available for 4243 men and 4722 women, of whom 303 and 73 died from IHD, respectively. In the logistic regression model the probability of IHD death for men was: $1 /(1+\exp (12.73-0.108 \times$ age -0.806 $\times$ smoking $-0.021 \times$ diastolic blood pressure$0.384 \times$ cholesterol $))$. The probability of IHD death for women was: $1 /(1+\exp (14.90-0.104$ $\times$ age $-1.24 \times$ smoking $-0.0306 \times$ diastolic blood pressure $-0.365 \times$ cholesterol)). All terms were statistically significant at a 0.001 risk level.

We then calculated the predicted probability of IHD death for each year the risk factor survey was conducted by entering the individual risk factor values observed in the survey into this logistic regression function and calculating the mean of these individual predicted risk scores. This procedure yielded the predicted changes in mortality since 1972 in each socioeconomic group, based on the changes in the risk factor levels in the consecutive surveys.

Data trends in IHD mortality in the socioeconomic groups were based on the death records for the years 1971-90, which were linked, by Statistics Finland, with the individual level records on occupation from the censuses in $1970,1975,1980$, and in $1985 .^{34}$ For the mortality analyses the data were pooled in five year periods after each census. Death rates were age standardised using five year age groups of the 1971-90 populations as a standard. The age standardised death rates and their 95\% confidence intervals were obtained using the Poisson regression model. ${ }^{11}{ }^{12}$

Occupation for the participants in the risk factor survey was obtained through linkage of 
Table 4 Observed (95\% confidence intervals) and predicted decline in age standardised IHD mortality by socioeconomic group. Data shown as percentages

\begin{tabular}{|c|c|c|c|c|c|c|}
\hline & \multicolumn{2}{|c|}{ White collar workers } & \multicolumn{2}{|c|}{ Blue collar workers } & \multicolumn{2}{|l|}{ Farmers } \\
\hline & Observed ${ }^{\star}$ & Predictedt & Observed ${ }^{*}$ & Predicted $t$ & Observed ${ }^{\star}$ & Predicted $f$ \\
\hline \multicolumn{7}{|l|}{ Men } \\
\hline $1971-75$ & - & - & - & - & - & - \\
\hline $1976-80$ & $24(10$ to 36$)$ & 14 & $7(-2$ to 15$)$ & 13 & 12 ( 2 to 22$)$ & 12 \\
\hline $1981-85$ & 32 (19 to 42$)$ & 26 & $19(11$ to 26$)$ & 28 & $20(10$ to 30$)$ & 28 \\
\hline $1986-90$ & 61 (53 to 68$)$ & 28 & 40 (33 to 45$)$ & 30 & 37 (27 to 45$)$ & 33 \\
\hline \multicolumn{7}{|l|}{ Women } \\
\hline $1971-75$ & - & - & - & - & - & - \\
\hline $1976-80$ & $24(-14$ to 50$)$ & 25 & 21 (1 to 38$)$ & 19 & $0(-28$ to 22$)$ & 23 \\
\hline $1981-85$ & $29(-4$ to 52$)$ & 27 & $32(14$ to 46$)$ & 29 & $23(-2$ to 42$)$ & 35 \\
\hline $1986-90$ & $57(34$ to 71$)$ & 41 & 43 (27 to 55$)$ & 35 & $20(-9$ to 41$)$ & 39 \\
\hline
\end{tabular}

* Observed decline after 1971-1975 based on the national cause of death data linked with census records. $\dagger$ Predicted decline based on logistic regression model and risk factor survey in 1972, 1977, 1982, and 1987.

records in the similar way as with the mortality data. Subjects were divided into three social classes on the basis of the 21 grade occupation variable in the population census data. The three social classes used were white collar workers, blue collar workers, and farmers. Pensioners were classified according to their past occupation. Housewives and other persons without present or past occupation were classified according to the head of the household. Employers and own account workers in other areas than agriculture, students, pensioners with missing past occupation, and others with missing data on socioeconomic status were excluded from the analyses.

\section{Results}

Diastolic blood pressure decreased by $4.5 \mathrm{~mm}$ $\mathrm{Hg}$ in men and by $9.1 \mathrm{~mm} \mathrm{Hg}$ in women from 1972 to 1987 . Serum total cholesterol declined by about $0.55 \mathrm{mmol} / 1$ in men and $0.83 \mathrm{mmol} / 1$ in women (table 2). These changes were not statistically significantly different between the socioeconomic groups. Also, the decreases in smoking among men were similar in the different socioeconomic groups. Smoking did not change among female white collar workers but increased among blue collar workers and farmers ( $p=0.04$ for the difference). Predicted mortality calculated by the logistic regression function declined similarly in all socioeconomic groups both for men $(\mathrm{p}=0.19)$ and for women $(\mathrm{p}=0.67)$.

Both in men and women IHD mortality was highest among blue collar workers during the study period (table 3 ). The relative differences between male white collar and blue collar workers increased over the time, but the greatest increase occurred during the 1980s. The relative difference between female white collar workers and blue collar workers remained about the same until the late 1980s.

The observed age standardised IHD mortality declined by $61 \%$ among white collar male workers, $40 \%$ among blue collar workers, and $37 \%$ among farmers (table 4 ). Based on the logistic regression model and observed risk factor changes, the predicted declines were $28 \%, 30 \%$, and $33 \%$, respectively. Hence, the risk factor changes among men explained $46 \%$ of the decline in IHD mortality among white collar workers, $75 \%$ among blue collar workers, and $89 \%$ among farmers. A particularly noticeable difference emerged in the $1980 \mathrm{~s}$ when the mortality in white collar workers declined by much more than what could be predicted by risk factor changes, while the decline in IHD mortality among blue collar workers and farmers essentially followed the decline predicted by the risk factors.

In women, the observed decline was $57 \%$ among white collar workers, $43 \%$ among blue collar workers, and $20 \%$ among farmers. The respective predicted declines were $41 \%, 35 \%$, and $39 \%$. Hence, the risk factor changes explained $72 \%$ and $81 \%$ of the observed declines among white and blue collar workers, but the decline among farmers was only half of that than predicted by the risk factor changes.

\section{Discussion}

Despite the large decline in IHD mortality in Finland, relative differences between the socioeconomic groups have been growing, especially among men. ${ }^{3}$ According to the present analyses the main reason for this widening gap has been that mortality from IHD declined more among male white collar workers in the 1980s than what could be predicted from the observed risk factor changes, while the risk factors among blue collar workers and among farmers predicted the observed decline quite well. There are several possible explanations for this. Coronary bypass surgery was introduced on a large scale in the $1980 \mathrm{~s}^{13}$ In the beginning there was not enough capacity in the public health care to do all the required operations and in the private sector the costs may have been too high for many blue collar workers. Mortality from recurrent infarctions has declined especially in the $1980 \mathrm{~s} .{ }^{13}{ }^{14}$ Secondary prevention in the form of counselling on risk factors has also improved during the 1980s. It may be so that the white collar workers have been more successful in changing their lifestyles after the infarction. The present regression model is very simple. It does not take into account other risk factors like physical activity, obesity, alcohol use, antioxidants, and other dietary changes affecting cholesterol level. Changes in these factors may be different between socioeconomic groups.

There are few methodological problems in this paper. IHD mortality has a large annual variation. We therefore used five year intervals to obtain sufficient stability for the analyses. The second uncertainty is how well we could measure the risk factor changes from 1972 to 
1987. Smoking was assessed using the same questions. Serum cotinine measurements in the 1992 survey indicated that self reported smoking is very realiable. Decline in the response rate over time may lead to an overestimation of smoking change. In blood pressure measurement, a short cuff was used in 1972 and 1977 and a longer one in 1982 and 1987. This may have led to the overestimation of decrease in blood pressure. Methodological difference of $2.4 \%$ in cholesterol measurement was corrected in the analyses. However, changes in blood pressure and cholesterol methodology were the same in all socioeconomic groups, and do not affect differences between them.

The third problem is the lag time between risk factor and mortality changes. We used risk factor levels shortly after the beginning of the five year period to estimate the predicted IHD mortality during each five year follow up. This was chosen on the basis of the results of cholesterol lowering trials, which suggest that the beneficial effects of cholesterol lowering on mortality are seen already after two years of treatment. ${ }^{15}$ Also the main benefits from quitting smoking are seen within five years. The predictive model used in our study was based on 15 year follow up of the 1972 cohort. Parameter estimates obtained from the later cohorts were fairly similar with those of the 1972 cohort, and therefore using them would not have had any practical effect on our prediction.

In conclusion, smoking, serum cholesterol, and blood pressure has decreased considerably in eastern Finland since the early 1970s. Decrease has been fairly similar in all socioeconomic groups. Decrease in IHD mortality has been greater among white collar workers than blue collar workers and farmers. Especially among male blue collar workers and farmers most of the mortality decrease could be predicted from the decrease in risk factors, but among white collar workers the decrease in mortality was larger than predicted by risk factor changes.

Conflicts of interest: none.

1 Marmot $M$. Coronary heart disease: rise and fall of a modern epidemic. In: Marmot M, Elliot P, eds. Coronary heart disease epidemiology: from aetiology to public health. Oxford: disease epidemiology: from aetiology to pub

2 Vartiainen E, Puska P, Pekkanen J, et al. Changes in risk factors explain changes in mortality from ischaemic heart disease in Finland. BMF 1994;309:23-7.

3 Valkonen T, Martelin T, Rimpelä A, et al. Socio-economic mortality differences in Finland 1981-1990. Helsinki: Statistics Finland, Population 1993; 1993:1

4 Valkonen $\mathrm{T}$. Trends in regional and socio-economic mortality differentials in Finland. International fournal of Health Sciences 1992;3:157-66.

5 Holme I, Helgeland A, Hjermann I, et al. Four-year mortality by some socioeconomic indicators: The Oslo Study. $\mathcal{F}$ Epidemiol Community Health 1980;34:48-52.

6 Marmot MG, Shipley MJ, Rose G. Inequalities in death-specific explanations of a general pattern? Lancet 984;i:1003-6.

7 Pocock SJ, Shaper AG, Cook DG, et al. Social class differences in ischaemic heart disease in British men. Landifferences in ischae

8 Rosengren A, Wedel H, Wilhelmsen L. Coronary heart disease and mortality in middle aged men from different occupational classes in Sweden. BMF 1988;297:1497-500.

9 Davey Smith G, Shipley MJ, Rose G. Magnitude and causes of socioeconomic differentials in mortality: further evidence from the Whitehall Study. F Epidemiol Community Health 1990;44:265-70.

10 Pekkanen J, Tuomilehto J, Uutela A, et al. Social class, health behaviour, and mortality among men and women in eastern Finland. BMF 1995;311:589-93.

11 Vartiainen E, Puska P, Jousilahti P, et al. Twenty-year trends in coronary risk factors in North Karelia and in other areas in coronary risk factors in North Karelia and

12 Payne C, ed. The GLIM system. Release 3.77, manual. Oxford: The Numerical Algorithms Group, Royal StatistiOxford: The Num

13 Salomaa VV, Miettinen H for the FINMONICA AMI Register Group. Incidence of and mortality from acute myocardial infarction in Finland in 1983-1992. Abstracts of the 35th Annual Conference on Cardiovascular Disease Epidemiology and Prevention. Circulation 1995;91:14.

14 Salomaa V, Arstila M, Kaarsalo E, et al. Trends in the incidence and mortality from coronary heart disease in Finland, 1983-1988. Am F Epidemiol 1992;136:1303-15.

15 Frick MH, Elo O, Haapa K, et al. Helsinki Heart Study: primary prevention trial with gemfibrozil in middle-aged men with dyslipidemia: safety of treatment changes in risk facwith dyslipidemia. safety of treatment, changes in risk fac1987;317:1237-45. 